\section{Participant recruitment to FiCTION, a primary dental care trial - survey of facilitators and barriers}

\author{
A. Keightley, ${ }^{* 1}$ J. Clarkson, ${ }^{2}$ A. Maguire, ${ }^{3}$ C. Speed ${ }^{4}$ and N. Innes ${ }^{1}$
}

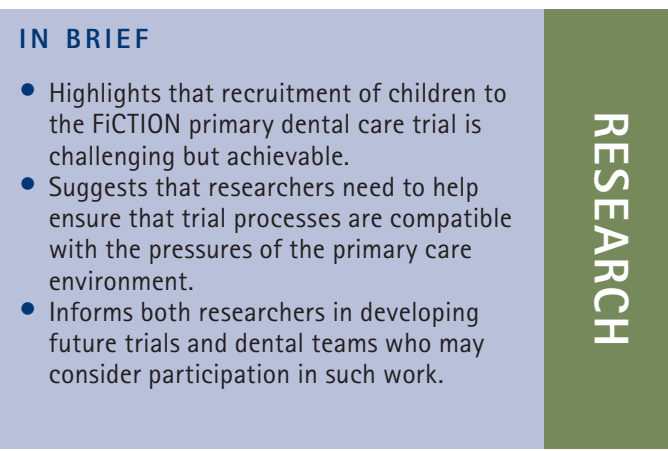

\begin{abstract}
Objective To identify reasons behind a lower than expected participant recruitment rate within the FiCTION trial, a multicentre paediatric primary dental care randomised controlled trial (RCT). Subjects (materials) and methods An online survey, based on a previously published tool, consisting of both quantitative and qualitative responses, completed by staff in dental practices recruiting to FiCTION. Ratings from quantitative responses were aggregated to give overall scores for factors related to participant recruitment. Qualitative responses were independently grouped into themes. Results Thirty-nine anonymous responses were received. Main facilitators related to the support received from the central research team and importance of the research question. The main barriers related to low child eligibility rates and the integration of trial processes within routine workloads. Conclusions These findings have directed strategies for enhancing participant recruitment at existing practices and informed recruitment of further practices. The results help provide a profile of the features required of practices to successfully screen and recruit participants. Future trials in this setting should consider the level of interest in the research question within practices, and ensure trial processes are as streamlined as possible. Research teams should actively support practices with participant recruitment and maintain enthusiasm among the entire practice team.
\end{abstract}

\section{INTRODUCTION}

The 'Filling children's teeth - indicated or not' (FiCTION) trial is a primary carebased multi-site, three-arm, parallel group, patient-randomised RCT (trial registration: ISRCTN77044005). ${ }^{1}$ It aims to determine which approach to the management of carious primary teeth is the most successful in the context of primary dental care in the UK. All trials estimate a target recruitment period - FiCTION started with 12 months, however, the rate of participant recruitment was lower than anticipated. The STEPS study, which investigated strategies for trial enrolment and participation, found only 31\% of randomised controlled trials (RCTs) recruited to time and target. ${ }^{2}$ A review by Treweek and colleagues recognised that achieving recruitment is an issue in medical research

\footnotetext{
'Dundee Dental School, Child Oral Health, Division of Oral Health Science, Dundee, DD1 4HN; ${ }^{2}$ University of Dundee, Dental Health Services Research Unit, Dundee, DD1 4HR; ${ }^{3}$ Newcastle University, Centre for Oral Health Research, School of Dental Sciences, Framlington Place, Newcastle upon Tyne, NE2 4BW; ${ }^{4}$ Newcastle Clinical Trials Unit, Institute of Health and Society, Newcastle, NE2 4BW

${ }^{*}$ Correspondence to: Mr Alexander Keightley Email: a.keightley@dundee.ac.uk
}

Online article number E22

Refereed Paper - accepted 25 July 2014

DOI: 10.1038/sj.bdj.2014.1009

${ }^{\circledR}$ British Dental Journal 2014; 217: E22 and that knowledge on how to increase recruitment at sites participating in a trial is currently lacking. ${ }^{3}$ This challenge was further reinforced by a recent survey of clinical trials units directors where 'research into methods to boost recruitment in trials' was found to be their highest research priority. ${ }^{4}$ The FiCTION trial involves the recruitment of children (aged 3-7 years), which the RECRUIT study found to be even more challenging. ${ }^{5}$ Additionally, these children are being recruited by more than 50 general dental practices across five regional centres over the UK. These general dental practices were already busy and few of them had participated in research before. ${ }^{6,7}$

Why attempt such a challenge? For the primary care dentist faced with the prospect of managing a child with caries, there is little direct evidence to guide their decisions. Existing evidence has shown that a number of approaches to the treatment of carious primary teeth can be successful. ${ }^{8}$ However, very few trials have compared the relative merits of these differing approaches in the context of primary dental care - the setting where children in the UK routinely receive their dental care. Despite improvements in child oral health, dental caries continues to affect 28-33\% of 5-year-olds in the UK, ${ }^{9,10}$ and this is a disease that can progress to cause excruciating pain and infection. ${ }^{11}$ The lack of clear answers to guide a dentist's decisions is reflected in the low care index with only $10 \%$ of decay in 5-year-olds currently restored (ft/dmft) in the UK..$^{9,10}$

FiCTION needs to recruit sufficient numbers of children to answer this important clinical question. With few large dental RCTs undertaken in primary dental care, little guidance was available on how barriers to participant recruitment could be overcome. It was important to ensure that, as a research team, we were doing everything possible to support FiCTION practices with participant recruitment. To understand the barriers to recruitment in medical studies, Kaur et al. developed a survey tool investigating recruitment in multi-centre medical RCTs. ${ }^{12}$ Using this tool, our aim was to identify reasons for FiCTION's lower than predicted participant recruitment rate and develop practical strategies to support practices in maximising future participant recruitment.

\section{METHODS}

The Kaur et al. survey tool was modified to reflect the context of the FiCTION trial; the major contextual themes being the dental practice setting, the primary care environment and recruitment of child participants. ${ }^{12}$ This modification process was undertaken by a team comprising; two clinical chief investigators, the senior trial manager and the clinical researcher and incorporated their 
experience and observations of participant recruitment in FiCTION. Among this group, multiple rounds of discussion were held until a consensus was reached on the final structure of the survey.

The survey tool was web-based with quantitative and qualitative sections. ${ }^{13}$ For the quantitative section, respondents were presented with a six point Likert scale, -3 for a strong barrier, to +3 for a strong facilitator (no 0 score), and were asked to rate 44 factors related to participant recruitment. These factors were grouped into six categories; trial features (nine factors), practice administration (four factors), child/ parent traits (12 factors), practice team (ten factors), family information and consent (four factors) and FiCTION research team (five factors). Factors that respondents had not experienced or considered not applicable to the recruitment process were rated as N/A.

The qualitative section contained five free-text boxes with open questions. Respondents were encouraged to express their experiences and to make suggestions to improve participant recruitment.

For the analysis of the quantitative section, a summary score for each of the 44 factors was calculated by totalling the number and strength of responses. A score of '7' could be achieved if three respondents rated a factor ' +3 ' and one respondent rated it ' -2 '. These summary scores for each factor were placed in ranked order, and factors scoring a summary score of more than +3 were considered facilitators, factors scoring less than -3 were considered barriers and those scoring between -3 and +3 were considered neutral factors.

For the analysis of the qualitative section, two investigators independently grouped the open responses into themes. The derived lists of themes and allocation of responses were compared and any areas of disagreement resolved through discussion and mutual agreement (NI and $\mathrm{AK}$ ).

\section{RESULTS}

There were 39 individual responses, which are broken down by the geographical FiCTION centre and practice team role in Table 1 ; for context the number of practices recruiting to the trial in each area is also given.

\section{Ranking of factors related to recruitment}

The overall scores that respondents gave to the 44 factors, categorised as facilitators, neutral and barriers are shown in Tables 2-4. In order to determine the relative influence of the six categories, a sum of all factor scores within each category was generated. The ranking of the sum scores were; FiCTION research team (267), practice team (171),

\section{Table 1 Responses by FiCTION Centre and practice team role}

\begin{tabular}{|c|c|c|c|c|c|}
\hline \multirow{2}{*}{$\begin{array}{l}\text { FiCTION Centre } \\
\text { (number of } \\
\text { recruiting practices) }\end{array}$} & \multirow{2}{*}{$\begin{array}{l}\text { Total number } \\
\text { of responses }\end{array}$} & \multicolumn{4}{|c|}{ Number of responses by practice team role } \\
\hline & & $\begin{array}{l}\text { Site lead/Principal } \\
\text { dentist }\end{array}$ & Dentist & $\begin{array}{l}\text { Dental } \\
\text { nurse }\end{array}$ & $\begin{array}{l}\text { Practice } \\
\text { manager }\end{array}$ \\
\hline $\begin{array}{l}\text { Scotland } \\
(n=9)\end{array}$ & 11 & 4 & 2 & 3 & 2 \\
\hline $\begin{array}{l}\text { North East England } \\
(\mathrm{n}=11)\end{array}$ & 8 & 3 & 0 & 3 & 2 \\
\hline $\begin{array}{l}\text { Yorkshire } \\
(n=7)\end{array}$ & 6 & 3 & 0 & 2 & 1 \\
\hline $\begin{array}{l}\text { Wales } \\
(n=4)\end{array}$ & 5 & 1 & 0 & 0 & 4 \\
\hline $\begin{array}{l}\text { London } \\
(n=9)\end{array}$ & 9 & 2 & 2 & 4 & 1 \\
\hline $\begin{array}{l}\text { Totals } \\
(n=40)\end{array}$ & 39 & 13 & 4 & 12 & 10 \\
\hline
\end{tabular}

Table 2 Overall scores for factors rated as facilitators to recruitment

\begin{tabular}{|c|c|c|}
\hline Category & Factor & $\begin{array}{l}\text { Overall } \\
\text { score }\end{array}$ \\
\hline 6 & Ease of ability to contact the FiCTION trial team & 77 \\
\hline 6 & Clarity and frequency of communication from the FiCTION trial team & 65 \\
\hline 6 & Frequency of evening meetings with other practices and local FiCTION trial team & 56 \\
\hline 6 & Motivation of the FiCTION trial team & 55 \\
\hline 4 & Interest in the particular research question within practice & 43 \\
\hline 1 & Trial publicity and merchandise & 40 \\
\hline 1 & Organisation of training day & 38 \\
\hline 4 & Importance of research generally in clinical practice & 36 \\
\hline 4 & Research experience of practice team & 32 \\
\hline 1 & Clinical equipoise & 28 \\
\hline 4 & Motivation of practice team & 27 \\
\hline 4 & Clinician attitude to involving patients in research & 27 \\
\hline 1 & Patient inclusion criteria & 24 \\
\hline 4 & Presence of designated research nurse/practitioner within practice & 19 \\
\hline 1 & Payments to practice to reimburse time & 15 \\
\hline 6 & Timely processing and payment of service support costs & 14 \\
\hline 4 & Research culture in local area & 9 \\
\hline 5 & Experience and training of clinical team in seeking consent & 5 \\
\hline 3 & Childs'/parents' attitude towards taking part in a clinical trial & 4 \\
\hline 4 & Clinician preference for particular treatment & 4 \\
\hline
\end{tabular}

trial features (136), family information and consent (-26) practice administration $(-45)$ and child/parent traits (-101).

\section{Themes from free-text responses}

The respondents were asked to give responses to five open questions. From the responses to these questions, the main themes are given illustrated by example quotes.

\section{Facilitators to recruitment}

Respondents were asked to identify the facilitators considered as being most important to participant recruitment; when analysed three themes developed:

Positive support from central FiCTION research team: 'Even though my recruitment numbers are small to date I have received excellent support and encouragement from the FiCTION trial team. There has not been 


\begin{tabular}{|c|c|c|}
\hline Category & Factor & Overall score \\
\hline 4 & Unfamiliarity in discussing research with patients & 3 \\
\hline 1 & Time required for collection of clinical data (that is, ICDAS) & 1 \\
\hline 3 & Additional trial investigations for children/parents & 0 \\
\hline 5 & Difficulty in approaching patients for consent & 0 \\
\hline 3 & Childs'/parents' familiarity with the clinical treatments involved & -1 \\
\hline 3 & Childs'/parents' concerns about a treatment new to them & -2 \\
\hline 3 & Language or cultural barriers among families & -2 \\
\hline 1 & Seasonal variation in patient attendance & -3 \\
\hline 1 & Clarity in presentation of trial information & -3 \\
\hline
\end{tabular}

\begin{tabular}{|c|c|c|}
\hline Category & Factor & $\begin{array}{l}\text { Overall } \\
\text { score }\end{array}$ \\
\hline 1 & Study protocol compared to usual clinical practice & -4 \\
\hline 2 & Time between training and opening recruitment at practice & -4 \\
\hline 2 & Time between opening recruitment at practice and achieving first recruit & -5 \\
\hline 3 & Trial questionnaires for children/parents & -6 \\
\hline 3 & Childs'/parents' preference for a particular treatment & -8 \\
\hline 2 & Recruitment target & -9 \\
\hline 5 & Time and setting of consent seeking & -10 \\
\hline 3 & Additional travel/time for children/parents & -11 \\
\hline 3 & $\begin{array}{l}\text { Impact of pre-existing social and emotional dynamics within families on discussion } \\
\text { of trial participation }\end{array}$ & -11 \\
\hline 3 & Childs'/parents' attitude to treatment choice by random allocation & -14 \\
\hline 3 & Duration of trial and follow up & -20 \\
\hline 5 & Amount and complexity of trial information provided & -21 \\
\hline 2 & Time to complete administrative work related to the trial & -27 \\
\hline 4 & Clinical workload & -29 \\
\hline 3 & Number of eligible children & -30 \\
\hline
\end{tabular}

undue pressure imposed as there is an understanding of our practice profile' - Site Lead (Scotland).

Involvement of full practice team: 'Strong practice team motivation and sheer number of invites coupled with parent willingness to participate' - Practice Manager (Wales).

Excitement of being involved in generation of new knowledge: 'Staff motivation, keen to make a difference to the future of dentistry' - Site Lead (London).

When asked to suggest how facilitative factors could be implemented across the trial, three themes arose:

Regular communication between central FiCTION research team and practices: 'They
- time taken for initial app [ointment], child gets bored' - Dental Nurse (Scotland).

Families response to being invited to participate in research: 'To [sic] much info that was sent to parents tended to frighten them off. Parents were more likely to attend during school holidays. The question about injections was the question most likely to 'frighten' them off' - Site Lead (North East).

Families ability to comprehend information about participating in research: Example 'Parents low level understanding' - Dentist (London).

Families established preferences about dental treatment: 'Parents/patients have a preferred treatment option. SSCs and no LA. Parents prefer dentist to make decision on best treatment option' - Site Lead (Scotland).

The additional burden research places on the family: 'Some eligible patients declined to take part because of the amount of paperwork involved' - Dental Nurse (North East).

Ensuring that practices are appropriately and promptly reimbursed for work done. 'Payment complex and not occurring' Practice Manager (North East).

When asked to suggest as to how barriers could be addressed, three themes emerged:

Additional efforts by practice to screen children: 'We have taken on a number of patients between the ages of 3-8 in the last few months. Patient who did not respond to a recall reminder we followed up ideally with a phone call asking patient if they would like to make appointment. If we were unable to contact by phone we sent a follow up letter' - Dental Nurse (North East).

Helping families understand the nature of the research: 'Had to explain to parents what they received. Some were confused what it meant' - Site Lead (North East).

Utilising additional diagnostic tests to find eligible children: 'Radiographs where appropriate when examining child patients' - Practice Manager (North East).

Suggestions to improve organisation of research in general dental practice

When asked to suggest how to improve participant recruitment in the context of general dental practice, five themes emerged:

Practices actively supported by FiCTION research team: 'Trial team very supportive. Parents very keen to be part of the trial' Practice Manager (North East).

Efforts by families and practice team is recognised and involvement is a rewarding experience: 'Children love it! Love the colouring in and merchandise' - Site Lead (North East).

Trial processes and paperwork simplified and streamlined: 'Simplify initial visit, too much paperwork, takes too long' - Dental Nurse (Scotland). 
Focus recruitment efforts on groups likely to be willing to participate: 'Regular attenders "trusted" what we told them about the trial' - Site Lead (North East).

Eligibility criteria inclusive as possible: 'Increase the age range.' - Dental Nurse (Yorkshire).

\section{Strategies for maximising recruitment}

These results identified a range of facilitators and barriers to recruitment, some of which related to inflexible elements of the trial. of the elements that could be modified at this stage of the trial, the following themes have been targeted to maximise the remaining recruitment period:

- Efforts by families and practice team are recognised - alongside promotional merchandise already distributed for participating children, practices that were successful in monthly recruitment were offered FiCTION branded mugs along with a tea break set. This limited gesture of thanks, recognising additional efforts practices make towards recruitment, was aimed at developing a positive atmosphere of fun and community among the practice teams

- Low numbers of eligible children encountered - recruitment of additional practices to increase the pool of children available for screening

- Involvement of full practice team the survey results formed the basis of open discussions with practices before committing to FiCTION, ensuring that newly recruited practices had as many of the positive features identified

- Training and delegation of tasks among full practice team - delivery of training was modified to maximise the practice team included, by delivering as much of it as possible within the individual practice

- Positive support from central FiCTION research team - close support from the FiCTION research team was offered to these new practices, particularly during the initial stages of setting up the key administrative processes.

\section{DISCUSSION}

For successful participant recruitment to a RCT within a typical general practice environment, our experience is that the whole practice team must be motivated towards it. The importance of this is reflected in the survey results that suggest that practice teams who believe that their additional hard work is worthwhile and contributes to important research of substantive relevance to their working lives, will commit the necessary effort. Respondents rated the FiCTION research question as a facilitator and while this will help promote recruitment, it can only achieve so much. The FiCTION research team has limited direct influence on the established practice team dynamics, however, the results of the survey identify the key avenues - communication/support from research team, dental team training, and gestures of thanks to recognise efforts to recruit to the trial.

Communication between the practice team and the FiCTION research team is important even before a practice becomes fully involved. While the local FiCTION clinical leads did utilise existing local knowledge and had discussions with practices before their involvement, there was no tool or exercise to formally evaluate a practice's ability to successfully recruit. This meant that any preexisting issues at practices did not become apparent until recruitment was due to begin. This survey has helped to guide recruitment of additional practices, informing open discussions with interested practices before them becoming fully involved in an attempt to pre-empt problems.

The survey highlighted numbers of eligible children as a barrier to recruitment. With the reported 28-33\% of 5-year-olds in the UK having obvious dental caries experience, it is initially counter-intuitive to suggest that this is a problem..$^{9,10}$ However, the distribution of caries in children is such that the obvious disease is carried by those with more severe disease, and, as a result, many of the 'easy' recruits have been found to be ineligible due to pain/sepsis. The use of bitewing radiographs in children has been shown to improve the detection of caries before it becomes clinically obvious. ${ }^{14}$ As such, radiographic examination presents a method of increasing the number of children found to be eligible at routine examination. The FiCTION trial protocol emphasises the use of radiographs in line with the UK FGDP guidelines, however, the low level of utilisation of bitewings in children is a known issue in primary dental care. ${ }^{15,16}$

Along with the numbers of eligible children, trial processes and paperwork was identified as a barrier to recruitment. Trial processes are standardised across research for good reason, ${ }^{17}$ but unnecessary complexity will sap motivation and every effort should be made to avoid overwhelming the practices. Before the main FiCTION trial a pilot rehearsal study was undertaken, which led to the refinement of many processes. ${ }^{18}$ For most practices, FiCTION is their first experience of being involved in a research trial requiring development of new skills. With a number of larger clinical trials now being undertaken in primary dental care, hopefully a pool of research capacity is being built for the future.

Regular communication between the research team and practices was highlighted as an important facilitator. As well as allowing for the transfer of knowledge, good communication allows practices to bring issues to the attention of the FiCTION research team. For example, it is important that practices are reimbursed service support costs (SSCs) in a timely and comprehensive manner. The Primary Care Trusts (PCTs), who had responsibility for such payments in England, were dissolved during the FiCTION recruitment period. Confusion among clinical commissioning groups, intended to assume PCT responsibilities, compounded the issue of payment of SSCs. While the FiCTION research team was not directly responsible, we pursued payments on behalf of FiCTION practices and kept practices informed.

Along with assisting practices with some of the bureaucratic challenges they have faced, the FiCTION research team provides practical support, such as assisting practices with setting up administrative processes to begin participant recruitment. Delivering support and training within the practice itself continues to be a useful method to ensure knowledge of FiCTION permeates the whole practice team. Participant recruitment is particularly reliant on administrative tasks. In general, these administrative tasks are assigned to dental nurses, receptionists and practice managers and therefore it is important that training is provided to the entire practice team.

We recognise that the trial sits in competition with many other demanding tasks in primary dental care. To help ensure that the trial remains prominent in the minds of practices, beyond our regular communication with practices, we have developed some limited gestures of thanks including local competition between practices, along with challenges to win trial branded items such as mugs. Initial reports from practices suggest that these gestures are appreciated and useful, primarily in introducing an element of 'fun' to the recruitment process, but also helping to keep trial recruitment prominent within the practice. ${ }^{5}$ Once participant recruitment to FiCTION is successfully completed we intend to evaluate the impact of the strategies developed from this survey on the overall process of recruitment.

This survey was developed to provide input to an ongoing recruitment process. There was no opportunity to undertake a piloting exercise to validate the questions and responses. It would have be interesting 
to investigate the recruitment issue from the perspective of families involved, but the practicalities precluded investigation of this area at present. In due course, this aspect will be investigated further as part of the qualitative component of the FiCTION trial itself. To encourage respondents to be open and honest about the recruitment process, no efforts were made to track completion to individual practices or team members, or to determine whether the response represented an individual or collective opinion. This has meant that it has been impossible to calculate an overall response rate.

A number of reviews have been undertaken on the topic of recruitment to RCTs. ${ }^{3,19,20}$ The evidence base drawn on by these reviews primarily originates from secondary care and is medical in nature. While there is a suggestion that some intervention may be effective in improving recruitment (that is, telephone reminders or financial incentives), the reviews consistently comment on the limited evidence base and the inability to draw any definitive conclusions. Within the context of primary dental care literature on this topic is limited. However, our results complement many of the findings from general dental practitioner (GDP) interviews in their views on research in the primary dental care setting. ${ }^{21}$ These interviews found the relevance of the research question to be important and that GDPs had concerns about the time and financial implication of participating in trials.

\section{CONCLUSIONS}

Undertaking this scale and complexity of RCT in the primary dental care setting is a novel experience and has highlighted the unique challenges this environment presents. However, if we are to meet the demand for RCTs to answer these research questions, then it is crucial that trials understand how to successfully engage with practice teams. This online survey tool was found to be useful in developing a profile of the features required of practices to successfully screen and recruit participants. This practice profile has been successfully utilised in the screening of additional practices to join this ongoing trial and at the time of writing over 60 practices across the UK are contributing to the recruitment of over 1,000 children. Our findings suggest that future trials in this setting should be cognisant of the inherent level of interest in the research question within primary care, as this can facilitate motivation to overcome the inevitable challenges involved with research. Primary care trials should strive to streamline research processes where possible to minimise further burden in an already time pushed environment, while provide adequate support from the central research team.

The current undertaking of large RCTs in UK primary dental care, such as FiCTION, has shown that it is feasible to answer fundamental clinical questions by undertake trials in this setting. The experiences gained from such trials ought to be utilised to help develop sustained capacity to answer further research questions in the primary care setting.

We would like to thank all the members of staff at the FiCTION practices who took the time to complete the survey. Thanks are also due to the University of Dundee Survey Team for managing the conversion of the survey into its online form and the collation of results and the FiCTION Clinical Lead Secretaries for liaising with the FiCTION practices to introduce them to the survey and encouraging its completion.

1. Innes N P, Clarkson J E, Speed C, Douglas G V , Maguire A. The FiCTION dental trial protocol--filling children's teeth: indicated or not? BMC Oral Health 2013; 13: 25.

2. Campbell M K, Snowdon C, Francis D et al. Recruitment to randomised trials: strategies for trial enrolment and participation study. The STEPS study. Health Technol Assess Winch Engl 2007; 11: iii, ix-105.

3. Treweek S, Pitkethly M, Cook J et al. Strategies to improve recruitment to randomised controlled trials. Cochrane Database Syst Rev 2010; 4: MR000013.

4. Smith C T, Hickey H, Clarke M, Blazeby J, Williamson $P$. The trials methodological research agenda: results from a priority setting exercise. Trials 2014; 15: 32

5. Shilling $V$, Williamson P R, Hickey $H_{\text {, Sowden }}$ Smyth R L, Young B. Processes in recruitment to randomised controlled trials of medicines for children (RECRUIT): a qualitative study. Health Technol Assess Winch Engl 2011; 15: 1-116.

6. Innes N P, Evans D J, Clarkson J E, Foley J I.

Obtaining an evidence-base for clinical dentistry through clinical trials. Prim Dent Care J Fac Gen Dent Pract UK 2005; 12: 91-96.

7. Hopper L, Morris L, Tickle M. How primary care dentists perceive and are influenced by research. Community Dent Oral Epidemiol 2011; 39: 97-104.

8. Ricketts D, Lamont T, Innes N P T, Kidd E, Clarkson $J$ E. Operative caries management in adults and children. Cochrane Database Syst Rev 2013; 3: CD003808.

9. Davies G, Neville J, Rooney E, Robinson M, Jones A, Perkins C. National Dental Epidemiology Programme for England: oral health survey of five-year-old children 2012. London: Public Health England, 2013.

10. Macpherson L, Ball G, Conway D I et al. National Dental Inspection Programme 2012- report of the 2012 detailed National Dental Inspection Programme of primary 1 children and the basic inspection of primary 1 and primary 7 children. Edinburgh: Scottish Dental Epidemiological Coordinating Committee, 2012.

11. Finucane $D$. Rationale for restoration of carious primary teeth: a review. Eur Arch Paediatr Dent 2012: 13: 281-292.

12. Kaur G, Smyth R L, Williamson P. Developing a survey of barriers and facilitators to recruitment in randomized controlled trials. Trials 2012; 13: 218 .

13. Bristol Online Surveys (BOS). Online information available at http://www.survey.bris.ac.uk/ (accessed August 2014).

14. Newman B, Seow W K, Kazoullis S, Ford D, Holcombe T. Clinical detection of caries in the primary dentition with and without bitewing radiography. Aust Dent J 2009; 54: 23-30.

15. Faculty of General Dental Practitioners. Selection criteria for dental radiography. 3rd ed. London: Faculty of General Dental Practitioners, Royal College of Surgeons of England, 2013.

16. Taylor G K, Macpherson L M. An investigation into the use of bitewing radiography in children in Greater Glasgow. Br Dent J 2004; 196: 563-568.

17. The Medicines for Human Use (Clinical Trials) Regulations 2004. Online regulations available at http://www.legislation.gov.uk/uksi/2004/1031/ contents/made (accessed August 2014).

18. Marshman Z, Innes N, Deery C et al. The management of dental caries in primary teethinvolving service providers and users in the design of a trial. Trials 2012; 13: 143.

19. Fletcher B, Gheorghe A, Moore D, Wilson S, Damery S. Improving the recruitment activity of clinicians in randomised controlled trials: a systematic review. BMJ Open 2012; 2: e000496.

20. Rendell J M, Merritt R D, Geddes J R. Incentives and disincentives to participation by clinicians in randomised controlled trials. Cochrane Database Syst Rev 2007: 2: MR000021.

21. Hopper L, Aspinall A, Mackie I C, Blinkhorn F A. The views of dentists on their participation in a primary care-based research trial. Br Dent J 2008; 205: E4. 